\title{
A Study of the Joint Advertising Channels
}

\author{
Ming LEI, Shuguang SUN, Dan YANG
}

Department of Management Science and Information System, Guanghua School of Management, Peking University, Beijing, China. Email: leiming@gsm.pku.edu.cn, sunshuguang@gsm.pku.edu.cn, yangdan@gsm.pku.edu.cn

Received July 22, 2009; revised September 1, 2009; accepted October 11, 2009.

\begin{abstract}
The study of joint advertising channels decision-making is a very difficult issue. It mainly focuses on ads investment game between a manufacturer and a retailer in a vertical supply chain. With the rise of the game theory, scholars have been starting to investigate this issue in the frame of game theory in recent years. The paper improves the existing models and introduces single-period and multi-period modified models. The paper obtains the closed-form solutions to the single-period model and the simulation results of the multi-period model. We also examine the implications of these results and obtain some insights into the real practice.
\end{abstract}

Keywords: Joint Advertising Channel, Game Theory, Simulation Method

\section{Introduction}

The cooperative advertising has been an important and interesting issue in the channel management literature. The paper adopts the frame of the two-level supply chain: the upstream firm (a manufacturer) and the downstream firm (a retailer). When pushing a new product into market, both the manufacturer and the retailer need to advertise. Meanwhile, the manufacturer will provide the retailer some incentives. In this paper, we investigate how much the manufacturer and the retailer invest on the ads respectively and how much the manufacturer should pay for the retailer as a subsidy.

Actually, the study of the cooperative advertising has a long history. However, prior to the application of game theory, the papers had been viewing this question as a pricing problem, not a game. The examples of such kind are Jeuland and Shugan, Moorthy, Ingene and Parry [1-3].

Currently, more and more scholars adopt the frame of game theory to study the cooperative advertising. All these studies differ from each other by the hypothesis on forms of the ads expense and subsidy rate and by the game type.

As for the form of the ads expense and subsidy rate, Dant and Berger assumed that the manufacturer provided the retailer a constant subsidy for per product sold [4]. Doraiswamy et al. also adopted such an assumption [5]. Under this hypothesis, the manufacturer charges a lower wholesale price. Bergen and John proposed that the manufacturer and retailer share the ads cost together [6]. Under this assumption, the manufacture compensates the retailer for the ads cost. However, they thought that the retailer chooses the total amount of ads. It is somewhat unreasonable because the manufacturer usually hold the leading position nowadays. In the study of Steffen, Simon and Georges, ads are divided into two different types: long and national, short and local. And it is also assumed that the manufacturer and retailer have the right to make ads of these two kinds. The manufacturer compensates the retailer for the ads costs via two constant subsidy rates, respectively [7]. But this assumption is a little complicated because the retailer is declined to make the ads of the first kind. Bergen and John distinguished between national ads and local ads [6]. Zhimin Huang and Susan distinguished between ads made by the manufacturer and ads made by the retailer. And they also assumed that the retailer is compensated for her ads investment [8].

Most papers adopt the complete information static or dynamic game models. For example, Zhimin Huang and Susan used Cournot and Stackelberg model, while other papers only adopted Stackelberg model where the manufacturer acts as the leader. Although the static game model is simple to deal with, the cooperative advertising has a long-term effect therefore a multi-period game is necessary [8].

The paper employs the dynamic programming method to study the multi-period dynamics model. Unfortunately, there are many limitations on the application of such method so few papers have adopted the multi-period dynamics model. Steffen distinguished the "long- term and national" ads from the "short-term and local" ads. This 
paper assumed that the ads of the first kind have a long-term effect: the ads affect the manufacturer's reputation and the market demand later. To solve the model, the paper adopted some simplified function forms therefore made the demand equation unconvincing [7].To obtain a better understanding of cooperative advertising in the frame of game theory, the paper provides a single-period and multi-period models, on the basis of Huang and Susan, and Steffen. We get a closed-form solution to the single-period model and simulate the multi-period model. In the end, the paper explains the results from economics perspective.

\section{Modeling}

\subsection{Single-Period Model}

On the basis of Huang and Susan, and Steffen, the paper introduces a game theory model of the joint ads $[7,8]$. This model is a Stackelberg model with the manufacturer as the leader.

\subsubsection{Players}

There are two players: the manufacturer and the retailer. The players are rational persons with complete information. The decision variables of the manufacturer are the ads investment $(b)$ and subsidy rate $(t)$. The decision variable of the retailer is her ads investment $(a)$.

The subsidy the manufacturer compensates for the retailer is . Therefore, the profit functions are:

$$
\begin{aligned}
& \pi_{m}=\rho_{m} Q-t a-b \\
& \pi_{r}=\rho_{r} Q-(1-t) a
\end{aligned}
$$

where $m$ and $r$ stand for the manufacturer and the retailer respectively, $\rho$ stands for the profit ratio subtracting the ads cost, and $Q$ is the sales volume.

\subsubsection{Relationship between Market Demand and Ads Investment}

On the basis of Huang and Susan, and Steffen, we assume that the demand function satisfies:

$$
Q(a, b)=\left(\alpha-\beta a^{-\gamma} b^{-\delta}\right) \sqrt{G}
$$

where $G$ is the reputation of the manufacturer, $q$ and $a$ are the ads investments of the manufacturer and retailer respectively, $t$ is the subsidy rate, and $\alpha, \beta, \delta, \gamma$ are all positive constants.

In addition, we assume that the reputation of the manufacturer satisfies the following dynamics equation:

$$
\Delta G=\lambda b-\mu G
$$

These two equations are explained as follows:

1) The demand function implies that the market demand is affected by two factors: the reputation and cur- rent ads investment. The maximum value of the current market demand is and can be affected by the reputation $[7,8]$. It is widely assumed that the marginal revenue of the ads decreases, so the paper does not adopt the linear function.

2) In the reputation dynamics equation, the paper adopts the form of exponential decay. The model of such kind is generally used in depicting decay trend [9-11].

\subsubsection{Payoffs}

It is reasonable to propose that the objective of the retailer is to maximize her current profits. Although the reputation of the manufacturer has a long-term effect, the retailer cannot gain profits brought by the long-term effect. Admittedly, to enhance the manufacturer's reputation will increase the long-term sale volume therefore benefit the retailer indirectly. However, the profit gained by the manufacturer far overweighs that gained by the retailer. Meanwhile, the manufacturer can take several measures to grab away these indirect profits gained by the retailer [12]. For example, the manufacturer can change retailers or increase the commission. So the payoff of the retailer is $\pi_{r}$.

The objective of the manufacturer is to maximize the payoff:

$$
\text { Max: } w=(1-\phi) \tau \Delta G+\phi \pi_{m}
$$

where $\tau$ is a positive constant, adjusting the units of the two terms in (5). The manufacturer is concerned about both the current profits and the reputation. The manufacturer can benefit from the current product sales and the enhancement of reputation: the enhancement of reputation can increase the product sales in future. So the manufacturer wants to maximize $G$ and $\pi$ simultaneously [13]. However, these two goals conflict because to increase $G$ means to advertise more therefore to reduce current profits. The paper introduces a weight factor $\phi$ to reconcile the conflict. And $\phi$ is exogenous in the paper. It is somewhat unreasonable. In the second model later in the paper, we discuss how the manufacturer selects the most proper $\phi$.

\subsection{Multi-Period Model}

In this multi-period model, there are still two parties: the manufacturer and the retailer and they are rational person with complete information. And similar to the singleperiod model, this model is a Stackelberg model with the manufacturer as the leader.

The hypothesis of the market demand, profit functions, reputation dynamic equation is the same with that of the single-period model. The only difference is that we need a footnote $\mathrm{n}$ to denote the $\mathrm{nth}$ period. For example, the reputation dynamics equation is 


$$
\begin{gathered}
\Delta G=\lambda b_{n}-\mu G_{n-1} \\
\text { or } \quad G_{n}=\lambda b_{n}+(1-\mu) G_{n-1}
\end{gathered}
$$

The goal of the retailer is still to maximize its profit at each period, i.e. $\pi_{r}(n)$. However, the goal of the manufacturer changes a little, for the long-term profit is in the consideration of the manufacturer.

\subsubsection{Goal of the Manufacturer}

We suppose that the goal of the manufacturer is

$$
\text { Max: } W=(1-\phi) \tau G_{N}+\phi \sum_{n=0}^{N-1}\left((1+\kappa)^{N-n-1} \pi_{m n}\right)
$$

where $n$ stands for the nth period, $k$ means the discounting rate, $\mathrm{N}$ is the total periods, $G_{N}$ stands for the long-term interests, $\phi \sum_{n=0}^{N-1}\left((1+\kappa)^{N-n-1} \pi_{m n}\right)$ is the current profits at the end of Nth period [13,14]. $\phi$ is the weight factor adjusting the units of the two terms in (6). Different from the single-period model, we do not introduce $\phi_{n}$ for $n$th period. Instead, the paper adopts an overall weight factor $\phi$ for the model. The manufacturer needs to choose $\phi_{n}$ for each period.

\subsubsection{Hypothesis of the Long-Term Strategies}

To make the model solvable, the paper introduces two hypotheses.

Hypothesis 1: Let $\omega$ be the weight factor for each period. And the N-period decision-making question can be converted into a question selecting the optimal $\omega$-path. What we need to do is just to solve the single-period optimization problem with the weight factor $\omega$ in each period. All these single-period solution under $\omega$-path consist the set $\Omega$. So another statement of this hypothesis is that if there is a solution sequence to question (6), the solution sequence must lie in the set $\Omega$.

This hypothesis is reasonable because it is naturally accepted that there exists a weight factor $\omega$ to adjust the long-term and short-term interests. Actually, this is our hypothesis in the first model. And this hypothesis is tenable in the management practice: if the long-term interests conflicted with the short-term interests, the mangers would allocate resources between them based on a weight factor.

The role of the hypothesis 1 is to convert a complicated multi-period optimization question into a route choice and single-period optimization questions. It is difficult to employ the dynamics programming method to solve the multi-period optimization question mathematically. That is why there are few papers that adopted the multi-period game theory model to deal with the cooperative ads question. It is true that some papers did adopt dynamics programming, but these papers made many unreasonable assumptions. In contrast, this paper does not sacrifice the reasonability for the simplification. We just confine the solution to the question into some forms. Fortunately, the hypothesis is reasonable from management practice perspective.

Hypothesis 2: the optimal $\omega$-path is monotonous and convergent to $\phi$.

This hypothesis is to restrict the types of the optimal $\omega$-path making the question solvable. It is reasonable to assume that the optimal $\omega$-path is convergent to $\phi: \omega$ and $\phi$ are two measures weighing the long-term and short-term interests. At the end of the multi-period game, the short-term measures should approximate the longterm measures. And when $\mathrm{N}=1, \omega$ must be equal to $\phi$.

Why do we assume that the optimal $\omega$-path is monotonous? In the real practice, how to choose $\omega$ depends on the strategy of the firm. $\omega$ means that the firm how to balance the market against profits. Generally speaking, there are three types of the strategies:

1) Market-leading strategy: in prophase, the goal of the firm is to sacrifice profits for expending market. So $\omega<\phi$ in prophase. In anaphase, $\omega$ Increases and approximates to $\phi$.

2) Profit-leading strategy: contrary to the marketleading strategy, the profit-leading strategy seeks profits in prophase. So $\omega<\phi$ at this stage, and $\omega$ decreases and approximates to $\phi$ in anaphase.

3) Equalitarian strategy: the firm has no preference towards profits or market. During the whole process, $\omega=\phi$.

Obviously, the hypothesis is justified under these three strategies.

\section{Analysis of the Models}

\subsection{Analysis of the Single-Period Model}

For this model, we can get the closed-form solution. We substitute (3) into (2) and then get the first order condition of the retailer:

$$
\begin{gathered}
\frac{d \pi_{r}}{d a}=\gamma \rho_{r} \beta \sqrt{G} b^{-\delta} a^{-(\gamma+1)}-(1-t)=0 \\
a=\left(\frac{\gamma \rho_{r} \beta \sqrt{G}}{(1-t) b^{\delta}}\right)^{\frac{1}{\gamma+1}}
\end{gathered}
$$

Therefore,

Next, we substitute (7) into (1) and get:

$$
\begin{aligned}
\pi_{m}= & \rho_{m} \alpha \sqrt{G}-\eta(1-t)^{\gamma /(\gamma+1)} b^{-\delta /(\gamma+1)} \\
& -\varepsilon t(1-t)^{-1 /(\gamma+1)} b^{-\delta /(\gamma+1)}-b
\end{aligned}
$$

where

$$
d w=(1-\phi) \tau \lambda d b+\varphi d \pi_{m}=0,
$$




$$
d w=(1-\phi) \tau \lambda d b+\phi d \pi_{m}=0 .
$$

The first order condition for the manufacturer is:

$$
d w=(1-\phi) \tau \lambda d b+\phi d \pi_{m}=0
$$

So, $b^{*}=\left[\left(\frac{\delta \phi}{\phi-\tau \lambda+\phi \tau \lambda}\right)^{\gamma+1} \gamma^{-\gamma} \beta\left(\rho_{m}-\gamma \rho_{r}\right) \sqrt{G}\right]^{1 /(\delta+\gamma+1)}$,

when $\tau \lambda<\frac{\phi}{1-\phi}$

$b^{*}=+\infty$, when $\tau \lambda \geq \frac{\phi}{1-\phi}$

$t^{*}=\frac{\rho_{m}-(\gamma+1) \rho_{r}}{\rho_{m}-\gamma \rho_{r}}$, when $\frac{\rho_{m}}{\rho_{r}}>\gamma+1$

$t^{*}=0$, when $\frac{\rho_{m}}{\rho_{r}} \leq \gamma+1$

We notice that when $(1-\phi) \tau \lambda$ is large enough $(\tau \lambda(1-\phi) \geq \phi)$, the manufacturer will increase the ads investment as much as possible. $1-\phi$ is the marginal value of the reputation and $\lambda$ is marginal reputation per ads. $\tau \lambda(1-\phi) \geq \phi$ means it is more profitable for the manufacturer to increase the ads investment. Therefore, the manufacturer will spare no effort to produce. $(12,13)$ demonstrate that the manufacturer will not compensate for the retailer as an incentive, until the marginal profit of the manufacturer is large and the marginal profit of the retailer is small $\left(\rho_{m} / \rho_{r}>\gamma+1\right)$, for the manufacturer benefits more from ads than the retailer does.

\subsection{Analysis of the Multi-Period Model}

As discussed above, the retailer holds the same behaviors as in the single-period model. As for the manufacturer, under the hypothesis 1 , the N-period optimization problem is converted into a problem selecting the optimal $\omega$-path and a series of single-period problems. And these single-period questions are almost the same with that in the first model. The only difference is that the parameter is $\omega$, rather than $\phi$.

Hence, the key part of the analysis of the multi-period model is how to select the optimal $\omega$-path. We will use the simulation method to solve this problem [13].

\subsubsection{Selecting the Optimal $\omega$-Path}

Selecting the optimal $\omega$-path is a part of the decision of the manufacturer. In the real practice, the manufacturer will calculate the profit $\mathrm{W}$ of the final period and then compares the profits of the all possible paths to select one to maximize $\mathrm{W}$. Now, we use computer programs to select the optimal $\omega$-path.
According to the hypothesis 2, the process of selection is divided into two steps:

Step 1: given the origin of the path, we try various approaching methods.

Step 2: select another origin, and repeat step 1.

As Figure 1 demonstrates, we experiment five different paths given two origins. They correspond to the three types of strategy respectively: the two paths above the horizontal line correspond to the profit-leading strategy; the two paths below the horizontal line correspond to the market-leading strategy; the horizontal line corresponds to the equalitarian strategy. The arrows mean changing the origins. Also as shown in Figure 1, we adopt two approximating methods: linear approximation and exponential approximation [13]. To be specific, the exponential approximation employs the function:

$$
\omega=\phi-\Delta \phi^{*} \frac{e^{-x}-e^{-1}}{1-e^{-1}}, \quad x=\frac{n}{N-1}
$$

Of course, these two approximation methods fail to cover all possibilities. However, we just want to find an approximate solution, not the precise solution. And the approximate solution is accurate enough for the real practice. If greater accuracy is needed, we can try more approximation functions and the origins.

\subsubsection{Parameters Valuating}

There are thirteen parameters: $\alpha, \beta, \gamma, \delta, \rho_{m}, \rho_{r}, \lambda, \mu$, $\phi, \tau, \kappa, N, G_{0}$. Their meanings and values in the simulation are demonstrated in the following Table 1.

These parameters can be divided into three groups:

Demand and profit: $\alpha, \beta, \gamma, \delta, \rho_{m}, \rho_{r}$

Market indicator: $\lambda, \mu$

Long-term strategy: $\phi, \tau, \kappa, N, G_{0}$

It is true that to get the best evaluation, we need to adopt the data in the real economy. However, there are too many limitations for us to do so. Another difficulty is that several parameters always change in the real practice, for example, $\rho$, but in the paper, they are viewed as constants.

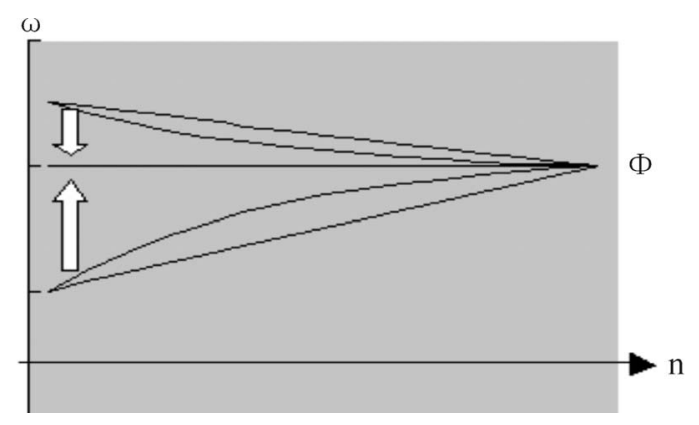

Figure 1. Various approaching methods 
Table 1. Parameters in the multi-period model

\begin{tabular}{ccl}
\hline parameter & value & implication \\
\hline$\alpha$ & 10 & market capacity \\
$\beta$ & 5 & ads effect coefficient \\
$\gamma$ & 0.5 & ads effect coefficient of the retailer \\
$\delta$ & 0.3 & ads effect coefficient of the manufacturer \\
$\rho_{m}$ & 0.18 & manufacturer's profit \\
$\rho_{r}$ & 0.1 & retailer's profit \\
$\lambda$ & 2 & reputation effect coefficient of the manufacturer \\
$\mu$ & 0.2 & reputation decay coefficient \\
$\phi$ & 0.8 & profit weight of the manufacturer \\
$\kappa$ & 0.06 & subsidy rate of the manufacturer \\
$\tau$ & 1 & conversion coefficient of reputation into profit \\
$\mathrm{N}$ & 10 & total periods of the game \\
$G_{0}$ & 10 & initial reputation of the manufacturer \\
\hline
\end{tabular}

Hence, we only can estimate them on the basis of the common sense and some constraints in the sense of mathematics and economics.

1) Ads investment is of medium term decision. Firms always make a decision every half or a year. The manufacturer's ads are national and large-scaled lasting one year; the retailer's ads are local and short-term lasting a quarter. Therefore, we assume that the each period lasts half a year.

2) The product life cycle is always 5 years so we assume that $\mathrm{N}=10 . \mathrm{N}$ is an important factor affecting the long-term strategy. We will relax the restrictions on $\mathrm{N}$ later in the paper.

3) The subsidy rate should be equal to the capital cost. We assume that the capital cost is $12 \%$. Because each period lasts half a year, the subsidy rate is $6 \%$ each period.

4) The ads effect coefficient of the retailer $\gamma$ is larger than that of the manufacturer $\delta$. It means that the retailer' ads have more influence in local market than the manufacturer's. The ads of manufacturer take effect in terms of reputation increase.

5) According to (12), $\rho_{m} / \rho_{r}>\gamma+1$ should hold.

\subsubsection{Optimal Long-Term Strategy:}

Now we study what long-term strategy the manufacturer should choose in the multi-period game and how the parameters affect the optimal long-term strategy. According to the classification of parameters, $\phi, \tau, \kappa, N, G_{0}$ affect the long-term strategy. In addition, $\lambda, \mu$ may influence the strategy because they affect the market demand.

By simulation, we find out that the game equilibrium paths of the market-leading and profit-leading strategies are as follows:
1) The equilibrium path of market-leading strategy;

2) The equilibrium path of profit-leading strategy.

With the evaluation of the parameters, the optimal strategy is the market-leading strategy (see Figure 2 and Figure 3 ). And we also adjust the value of the seven parameters that affect the long-term strategy slightly, the following results are gained:

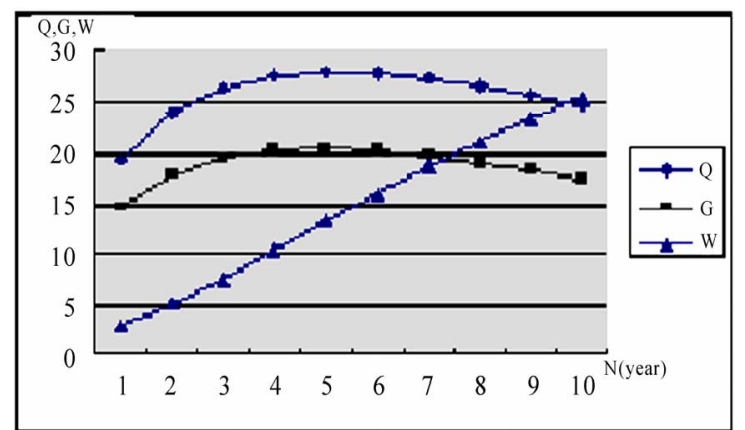

Figure 2. Demand, reputation, and manufacturer's profit (market-leading)

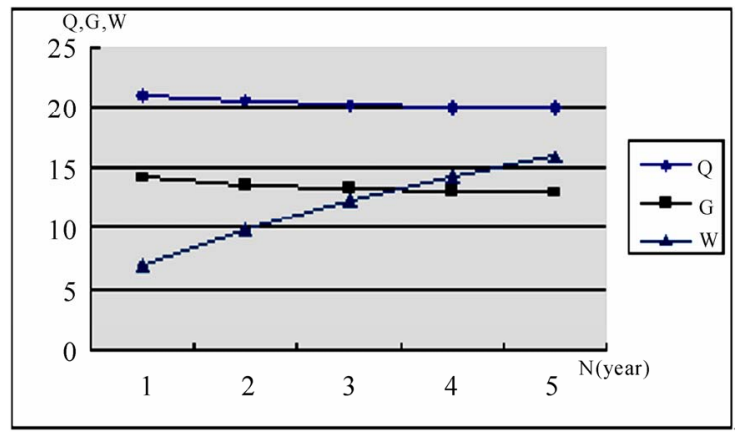

Figure 3. Ads and profit (market-leading) 


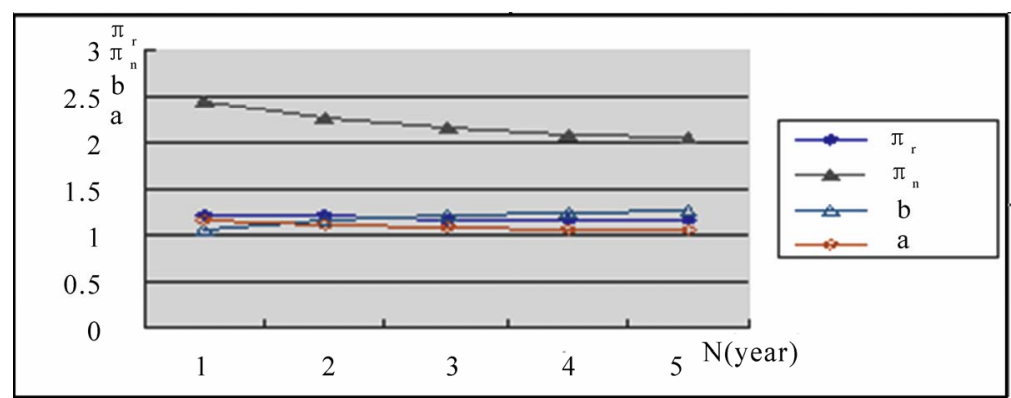

Figure 4. Demand, reputation, and manufacturer's profit (profit-leading)

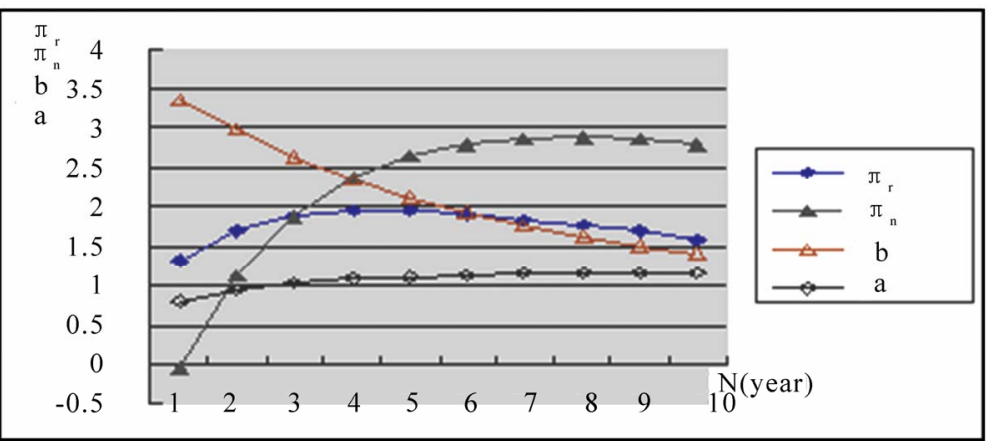

Figure 5. Ads and profit (profit-leading)

1) In most cases, the optimal strategy is the market-leading strategy.

2) In many cases where we had thought the profitleading strategy is dominant, it proves not to be the optimal, although the final profit $\mathrm{W}$ is almost the same.

3) In few cases where the profit-leading strategy is dominant (see Figure 4, Figure 5), the value of $\mathrm{N}$ is small.

These conclusions seem a little astonishing. However, there are indeed profound implications behind them. In the next section, we will discuss these results in detail.

\subsubsection{Parameters' Effect on Game Equilibrium}

In this section, we study the parameters $\gamma, \delta, \rho_{m}$, $\rho_{r}, \lambda, \mu$ how to affect the game equilibrium when the manufacturer operates according to the optimal strategy.

The following are the results of the simulation, where the values of $a, b, t, \pi_{m}, \pi_{r}$ are mean values of all periods. The value of $\mathrm{W}$ is calculated at the final period. The benchmarks are from Table 2 .

In the process of simulation, we find out that if a parameter affects the game equilibrium, the effect is monotonous. So we only experiment those cases with increasing values of the parameters.

\section{Discussion}

Now we explore the implications of the results above and get some suggestions on marketing management. We ne- ed to point out that the assumptions on profit functions, demand functions, and ads enhancement equation are the same in the single-period model and the multi-period model. These two models take the long-term effect of ads into consideration. So we can gain some results about the sales profit ratio and ads long-term effect.

\subsection{Sales Profit Ratio and Ads Long-Term Effect}

In the models of this paper, the retailer's ads have only short-term effect and the effect coefficient is $\gamma$; the manufacturer's short-term ads coefficient is $\delta$. The sales profit ratios of the retailer and manufacturer are $\rho_{r}$ and $\rho_{m}$ respectively.

According to the closed-form solution (7) and (10,11) to the single-period model, the short-term ads effect coefficients ( $\gamma$ and $\delta$ ) do not have a monotonous influence on the ads investments of the manufacturer and retailer. When the ads have more influence on the sales profits, the more ads will be made. Simultaneously, the more the ads affect the sales profits, the less ads investment is needed to achieve the given goal. Table 2 shows that the ads investment of the retailer and manufacturer ( $a$ and $b$ ) increase in $\gamma$ and $\delta$.

The manufacturer's sales profit ratio $\rho_{m}$ is positively correlated with her ads investment. This relationship is supported by the closed-form solution $(10,11)$ 
Table 2. Parameters' effect on game equilibrium

\begin{tabular}{|c|c|c|c|c|c|c|}
\hline & $a$ & $b$ & $t$ & $\pi_{m}$ & $\pi_{r}$ & $W$ \\
\hline Benchmark & 1.07 & 2.17 & 0.23 & 2.22 & 1.75 & 25.50 \\
\hline$\gamma=0.6$ & 1.13 & 1.92 & 0.17 & 2.35 & 1.54 & 26.64 \\
\hline$\delta=0.4$ & 1.01 & 2.70 & 0.23 & 2.48 & 2.23 & 28.62 \\
\hline$\rho_{m}=0.2$ & 1.18 & 2.38 & 0.33 & 2.81 & 2.01 & 31.76 \\
\hline$\rho_{r}=0.11$ & 1.04 & 2.11 & 0.12 & 2.29 & 1.85 & 26.20 \\
\hline$\lambda=2.2$ & 1.16 & 3.43 & 0.23 & 2.74 & 2.68 & 30.76 \\
\hline$\phi=0.85$ & 1.04 & 1.30 & 0.23 & 1.87 & 1.10 & 22.34 \\
\hline$\mu=0.3$ & 0.99 & 2.03 & 0.23 & 1.61 & 1.39 & 18.17 \\
\hline
\end{tabular}

and the Table 2. When the sales profit ratio increases, the manufacturer will sell more products and therefore make more ads. From the simulation results, we find out that the retailer's ads investment increases in the sales profit ratio. It is because the increase of the sales profit ratio leads to the increase of the compensation for the retailer's ads and hence to increase the retailer's ads investment.

Now, we turn our attention onto the subsidy rate. According to the closed-form solution $(12,13)$ to the single-period model, $t=1-1 /\left(\rho_{m} / \rho_{r}-\gamma\right)$. So $t$ increases in $\rho_{m} / \rho_{r}$ and decreases in $\gamma$. It is to say: the smaller the manufacturer's sales profit ratio is compared with the retailer's sales profit ratio and the larger the retailer's ads effect coefficient is, the lower the manufacturer will compensate for the retailer. This conclusion is also supported by the simulation results of the multiperiod model. The conclusion can be explained as follows:

The product sales benefit two players therefore they have incentives to advertise to increase the product sales. However, their profit-cost structures are different. The player (the manufacturer) who benefits more from ads wants another player (the retailer) to advertise more by taking measures to spur her. When the difference between their sale profit ratios becomes larger, the manufacturer will pay more for the retailer. And if the retailer has a large ads effect coefficient, she does not need many ads to achieve her sales goal or the retailer has strong incentives to advertise. So the manufacturer will need not to pay much to the retailer.

The closed-form solution (13) also shows an exception: when $\rho_{m} / \rho_{r} \leq \gamma+1$, the manufacturer will not pay for the retailer. It is because in this situation, the retailer gains a high profit or is expert in advertising. So the retailer may pay for the manufacturer conversely.

Consequently, the ads investment of the manufacturer is positively correlated with her sales profit ratio. The ads investment of the retailer is positively correlated with her sales profit ratio and the subsidy rate from the manufacturer. The subsidy rate is positively correlated with the quotient of the two sales profit ratios and negatively correlated with the retailer's ads effect coefficient.

\subsection{Reputation}

In the models, the reputation functions via enhancing the market capacity indirectly and the reputation is affected by the ads. The relevant variables are $\lambda, \mu, 1-\phi$.According to the solution $(10,11)$ to the single-period model, $\phi$ affects the manufacturer's ads monotonously: the increase of $\phi$ will lead to the decrease of the ads investment of the manufacturer $b$. This relationship is also shown in the simulation solution to the multi-period model: when $\phi$ increases from 0.8 to $0.85, b$ decreases from 2.17 to 1.30 . It is because $\phi$ is the weight factor of profit and profit conflicts with the market which needs ads support. If the manufacturer wants to increase the current profit, the investment for the long-term ads must be reduced.

As shown above, when $\lambda$ becomes larger, the manufacturer will advertise more. This conclusion is supported by the solution $(10,11)$ to the single-period model and the simulation results of the multi-period model: when $\lambda$ increases from 2.0 to $2.2, b$ increases from 2.17 to 3.43 . This result is explained as follows:

The manufacturer's ads have two effects: enhancing current product sales and enhancing the reputation and therefore promoting future sales. In the models, these two functions do not conflict. When $\lambda$ becomes larger, the manufacturer can gain more reputation. On the other hand, the current ads effect stays the same. Hence the manufacturer will advertise more.

The parameter $\mu$ is the decay coefficient of the reputation. From the simulation results of the multi-pe- 
riod model, it is found out that when $\mu$ increases, the average profits of the manufacturer and retailer decrease. It is because the market increases slower or decays faster in this situation.

According to (11), when $(1-\varphi) \tau \lambda \geq \varphi$, the manufacturer will advertise as much as possible. Why does it happen? $(1-\phi) \tau$ is the marginal profit of the reputation and $\lambda$ is the marginal reputation of the ads, so $(1-\phi) \tau \lambda$ is the marginal value of the ads. Meanwhile, $\phi$ is the marginal value of profit. The inequality $(1-\varphi) \tau \lambda \geq \varphi$ means that ads can bring the manufacturer more value than current profits do.

\subsection{Long-Term Game Strategy}

The long-term game strategy is a concept that the paper introduces to explain the simulation results of the multi-period model. This concept is employed to determine the weight factor of profit each period.

Generally speaking, there are several variables to affect the long-term strategy: $\mu, \phi, \kappa, N, G_{0}$. However, it seems that only the variable $N$ affects it according to the simulation results. Actually, the market-leading strategy is called the market penetration strategy and profit-leading strategy is called market skimming strategy. According to the marketing theory, if a firm plans to develop in long term, the market penetration is optimal. The firm should sacrifice profit in prophase to increase the market share, or else, the firm adopts the policy of "high price and high profit" in prophase and then withdraws from the market. Of course, there are no metaphase or anaphase plans in this situation.

That is why the optimal long-term strategy is always the market-leading strategy in the multi-period model when $N$ is large. There is no market withdrawal mechanism in the models. So when the game duration is long, the manufacturer has to adopt the market-leading strategy. This analysis justifies assumptions and the simulation method in models.

\section{Conclusions}

In this paper, we explore the interactions of ads investments between the manufacturer and retailer. Two types of game theory are employed to achieve our goal. In the first model: single-period model, we get a closed-form solution while in the multi-period model, we have to resort to the simulation methods to get some numerical solutions. The paper provides some interesting conclusions. The manufacturer's reputation has influence on the market demand and it is affected by the long-term ads investment only by the ads investment of the manufacturer, while the increase of the reputation is beneficial to the retailer too. At the same time, the manufacture must pay for the retailer to spur her ads investment. In such a context, the interaction between these two firms is complex.

The main results of the paper are:

1) The manufacturer's ads investment is positively correlated with her sales profit ratio and the retailer's ads investment is positively correlated with her sales profit ratio and the subsidy rate from the manufacturer.

2) The smaller the manufacturer's sales profit ratio is compared to the retailer's sales profit ratio and the larger the retailer's ads effect coefficient is, the lower the manufacturer will compensate for the retailer.

3) The increase of the manufacturer's profit weight and will lead to the decrease of the ads investment of the manufacturer, while as reputation effect coefficient becomes larger, the manufacturer will advertise more.

4) When the game duration is long, the manufacturer will adopt the market-leading strategy.

\section{Future Study}

\subsection{Improving the Models}

In this paper, we adopt the simulation method that imposes little limitation on modeling. We can introduce more complicated models: 1) there are more than two players; 2) the assumptions on the long-term ads effect are a little simple: attributing all long-term effect factors to the manufacturer's reputation. The future work can take other factors into consideration; 3) the future researches can introduce the information asymmetry into models. For example, the manufacturer does not know the sales profit ratio and the ads effect coefficient of the retailer, or the retailer knows little about the product quality and after service of the manufacturer.

\subsection{Applying the Models and Simulation into Specific Situations}

The simulation relies heavily on the valuation of the parameters. If we can get data from the firms in real practice and then simulate on the basis of the data, the results will be more convincing. The implications of these new results will bring us several new findings.

\section{REFERENCES}

[1] A. P. Jeuland, and S. M. Shugan, "Managing channel profits," Marketing Science, Vol. 2, No. 3, pp. 239-272, 1983.

[2] K. S. Moorthy, "Managing channel profits: Comment," Marketing Science, Vol. 6, No. 4, pp. 375-379, 1987.

[3] C. A. Ingene and M. E. Parry, "Channel coordination when retailers compete," Marketing Science, Vol. 14, No. 4, pp. 360-377, 1995.

[4] R. P. Dant and P. D. Berger, "Modeling cooperative ad- 
vertising decisions in franchising," Journal of the Operational Research Society, Vol. 49, No. 9, pp. 1120-1136, 1996.

[5] K. Doraiswamy, T. W. McGuire, and R. Staelin, "An analysis of alternative advertising strategies in a competitive franchise framework," Proceedings of Educator's Conference of American Marketing Association, pp. 463467, 1979.

[6] M. Bergen and G. John, "Understanding cooperative advertising participation rates in conventional channels," Journal of Marketing Research, Vol. 34, No. 3, pp. 357369, 1997.

[7] S. Jørgensen, S. P. Sigue, and G. Zaccour, "Dynamic cooperative advertising in a channel," Journal of Retailing, Vol. 76, No. 1, pp. 71-91, 2000.

[8] Z. Huang and S. X. Li, "Co-op advertising models in manufacturer-retailer supply chains: A game theory approach," European Journal of Operational Research, Vol. 135, No. 3, pp. 527-544, 2001.

[9] Deal and R. Kenneth, "Optimizing advertising expenditures in a dynamic duopoly," Operations Research, Vol.
27, No. 4, pp. 682-692, 1979.

[10] Chintagunta, K. Pradeep and D. Jain, "A dynamic model of channel member strategies for marketing expenditures," Marketing Science, Vol. 11, No. 2, pp. 168-188, 1992.

[11] Chintagunta and K. Pradeep, "Investigating the sensitivity of equilibrium profits to advertising dynamics and competitive effects", Management Science, Vol. 39, No. 9, pp. 1146-1162, 1993.

[12] K. C. Sen, "Advertising fees in the franchised channel," Journal of Marketing Channels, Vol. 4, No. 1, pp. 83-101, 1995.

[13] S. P. Wang, K. L. Judd and C. Gung, "Solving a savings allocation problem by numerical dynamic programming with shape-preserving interpolation," Computers \& Operations Research, Vol. 27, pp. 399-408, 2000.

[14] L. Tesfatsion, "Introduction to the special issue on agentbased computational economics," Journal of Economic Dynamics and Control, Vol. 25, No. 3-4, pp. 281-293, 2001. 